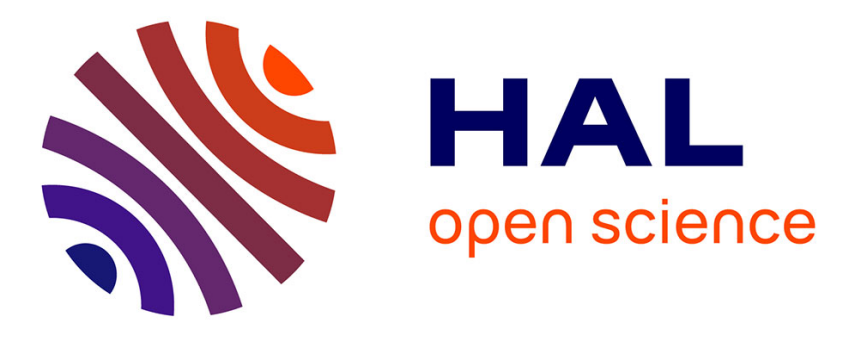

\title{
A Conventional Dialogue Model Based on Dialogue Patterns
}

\author{
Guillaume Dubuisson Duplessis, Alexandre Pauchet, Nathalie Chaignaud, \\ Jean-Philippe Kotowicz
}

\section{- To cite this version:}

Guillaume Dubuisson Duplessis, Alexandre Pauchet, Nathalie Chaignaud, Jean-Philippe Kotowicz. A Conventional Dialogue Model Based on Dialogue Patterns. International Journal on Artificial Intelligence Tools, 2017, 26 (01), pp.1760009. 10.1142/S0218213017600090 . hal-02121702

\section{HAL Id: hal-02121702 \\ https://hal.science/hal-02121702}

Submitted on 13 Mar 2021

HAL is a multi-disciplinary open access archive for the deposit and dissemination of scientific research documents, whether they are published or not. The documents may come from teaching and research institutions in France or abroad, or from public or private research centers.
L'archive ouverte pluridisciplinaire HAL, est destinée au dépôt et à la diffusion de documents scientifiques de niveau recherche, publiés ou non, émanant des établissements d'enseignement et de recherche français ou étrangers, des laboratoires publics ou privés. 


\title{
A Conventional Dialogue Model based on Dialogue Patterns
}

\author{
AUTHORS' DRAFT \\ Guillaume Dubuisson Duplessis Alexandre Pauchet
Nathalie Chaignaud Jean-Philippe Kotowicz
}

December 15, 2016

\begin{abstract}
Our work aims at designing a dialogue manager dedicated to agents that interact with humans. In this article, we investigate how dialogue patterns at the dialogue act level extracted from Human-Human interactions can be fruitfully used by a software agent to interact with a human. We show how these patterns can be leveraged via a dialogue game structure in order to benefit to the dialogue management process of an agent. We describe how empirically specified dialogue games can be employed on both interpretative and generative levels of dialogue management. We present DoGMA, an open-source module that can be used by an agent to manage its conventional communicative behaviour. We show that our library of dialogue games can be used into DoGma to generate fragments of dialogue that are strongly coherent from a human perspective.
\end{abstract}

\section{Introduction}

In heterogeneous multi-agent systems and mixed communities, software agents interact with one another and with humans. The range of artificial interactive agents is wide: autonomous interface agents[1], embodied conversational agents[2], intelligent virtual agents[3], mixed-initiative assisting agents[4], sensitive artificial listeners[5], socially adaptive agents [6] and so on[7]. As humans have the remarkable ability to communicate, reason and understand each other, interactive agents need to adapt their behaviour to human standards in order to ease the comprehension. When designing an interactive agent, dialogue management is a key feature $[4,8,9]$ since dialogue is an efficient and natural way of communicating with humans. However, designing the communication model of an interactive agent is challenging[3] and often leads to the integration of rigid dialogue management processes, such as a keyword spotter.

Dialogue is a joint and opportunistic activity[10]: the interlocutors coordinate their contributions to co-construct and co-control the dialogue. To model 
dialogue with an overall coherence, high-level structures have to be considered (e.g., formalising utterances according to their semantic content). One striking observation in human dialogue is the presence of recurrent patterns. They correspond to sequences of utterances that are frequently reoccurring (e.g., a question/answer pair)[11, 12]. We share the point of view of Orkin et al.[13, 14] that recurrent dialogue patterns occurring in Human-Human $(\mathrm{H}-\mathrm{H})$ interaction can be exploited to model Human-Machine (H-M) interaction.

In previous work[15, 16], we have presented our long-term goal to build a mixed-initiative assistant for information retrieval for the CISMEF system[17]. To this end, we have also described a data-driven methodology dedicated to the extraction of dialogue patterns[18], as well as its implementation from corpus collection to the formalisation of dialogue games from dialogue patterns[16]. This article (which is an extended version of [19]) describes the next step, i.e. how an interactive agent can employ a set of dialogue games to manage its conventional communicative behaviour. These dialogue games constitute the basic interaction units manipulated by the deliberative process of an interactive agent. We point out how these structures can be fruitfully exploited during the dialogue management process. We describe our open-source software DoGMA, designed as a module included in a dialogue manager. Finally, we present promising results from the first version of our system. We show how empirically specified dialogue games implemented into DoGMA generate fragments of interaction that are strongly coherent from a human perspective.

Section 2 draws some links with related work, with a focus on dialogue patterns and dialogue management. Section 3 presents a brief description of our data-driven methodology. Section 4 describes our dialogue game framework used to formalise interaction patterns. Section 5 shows how dialogue games can concretely and fruitfully be used during the dialogue management process of an interactive agent. We present DogmA, the dialogue game manager we propose, that implements a library of empirically specified dialogue games. Section 6 deals with the evaluation of the dialogue games in terms of coherence and naturalness. It also discusses the contribution of the model. Lastly, Section 7 concludes this article.

\section{Related Work}

Human dialogues contain regularities, such as ordered sequences of utterances, that can be considered as dialogue or interaction patterns $[11,12,18,20]$. In

dialogue modelling, interaction patterns have been analysed as an evidence of a plan that the interlocutors follow (plan-based approaches), or as the result of conventions that the dialogue participants (DPs) commit to (conventional approaches).

Plan-based approaches focus on the intentional structure of the dialogue[21]. Basically, these approaches consider that each speaker's utterance conveys an intention which is part of a global plan. The main idea is that DPs infer the underlying plan behind an utterance to produce a cooperative response. DPs have 
to infer the interlocutor's intentions, reason about it and produce a response accordingly to the underlying plan, rather than just to the speaker's previous utterance. These approaches have led to influential systems such as TRAINS[22] or Collagen[23, 24]. While these seminal works are limited to a specific domain, recent efforts aim at improving the adaptability of agents to multiple domains and subtasks[25, 26]. These approaches involves the recognition of complex intentions to provide agents with the ability of task assistance across multiple domains. Besides, recent works aims at improving semantic understanding techniques, e.g., by automatically inducing and filling dialogue slots[27] or by automatically discovering user intents and their associated arguments from in-domain and out-of-domain user utterances[28]. However, some weaknesses have been spotted for the plan-based approaches. Namely, the plan-recognition process remains a complicated task, technically difficult to set up[29]. Next, dialogue is an opportunistic activity[10] and consequently some sentences cannot be planned (e.g., clarifications)[30].

On the other hand, conventional approaches consider patterns as the result of DPs' efforts to follow human conventions. This point of view is based on the fact that many utterances seem to be conventionally triggered by the context (e.g., greetings) rather than planned. This has lead to models describing admissible sequences of utterances without a focus on the underlying intentions. Reoccurring patterns have been studied either in terms of dialogue grammars [31] or dialogue games[20,32].

These two approaches are often viewed as opposite, although some researchers argue that they are in fact complementary $[12,33,34,35]$ : communication processes are opportunistic joint actions between DPs[10]. The main characteristic of a joint action is the coordination between several people in order to produce participatory actions. Nevertheless, DPs cannot deliberate indefinitely during a dynamic activity such as dialogue, and coordination must thus stand on devices such as conventions reflected by interaction patterns. Dialogue can therefore be considered as a shared and dynamic activity that requires both high-level deliberative reasoning processes and low-level reactive responses.

In this article, we consider dialogue games as a structure that can be exploited to describe and explain human dialogue, to generate artificial dialogues dedicated to humans[20], and to manage human-agent interaction. A dialogue game is a bounded joint activity with an entry and an exit during which participants have a role (initiator or partner). The rules of the dialogue game specify the expected moves for each DP. Participants of a dialogue game are expected to play their roles by making moves according to the current stage of the game. The purpose of these games is to capture the conventions of human interaction. To the best of our knowledge, dialogue games have received only few attention from practical applications in the human-computer interaction field. On a theoretical level, dialogue games have been seen as initiativeresponse units[12], and as structures capturing commitments created during dialogue[33]. On both practical and formal level, rules of dialogue games have been represented as recursive transition networks for virtual agents[36, 37] and human-robot interaction[38, 39, 40]. As other researchers[12, 33], we propose to 
use a hybrid reactive/deliberative architecture where a theory of joint actions may serve as a "semantics" to the interaction patterns described as dialogue games.

\section{Data-Driven Methodology}

Our work is based on a data-driven methodology $[18,15,41]$ that is similar to the methodology used by other researchers[13]. It is a generalisation of the classic approach used to study corpora in various research fields such as linguistic psychology, and can be broken down into a sequence of steps: collection of a $\mathrm{H}-\mathrm{H}$ corpus, annotation of interaction units, pattern extraction and modelling. This methodology aims to extract dialogue patterns in order to enrich high-level structures, useful for the dialogue management process of an agent interacting with a human.

Our long-term goal is to build a mixed-initiative assistant for information retrieval for the CISMEF system[17]. To that end, we presented an implementation of our methodology from corpus collection to pattern extraction in previous work[16]. A thorough description can be found in[35]. This application took place on a task-oriented corpus involving collaborative information search between an expert and a novice user, performed on a medical search engine. Dialogues were recorded during the task where the expert and a user were facing a computer using the advanced search interface. This experiment produced 18 dialogues between 2 experts and 18 users. It contains approximately 33,000 words and 1,054 turns. The annotation step was performed with the DIT++ annotation scheme of communicative functions[42]. 5,484 functional segments and 6,343 communicative functions were produced. The extraction process was carried out on $2 / 3$ of our initial corpus (the other third constitutes the reference corpus used in our validation).

All in all, we formed a database of task-oriented dialogue games available to an interactive agent. It contains 8 items: 3 action-oriented dialogue games and 5 information-seeking-oriented dialogue games ${ }^{1}$. They mainly formalise initiativeresponse patterns consisting in adjacency pairs with preferred and dis-preferred second pair parts[43] (see, e.g., turns 8 and 9 in table 2). We have shown that this database provides a suitable coverage of $\mathrm{H}-\mathrm{H}$ interaction patterns appearing in the reference corpus[16].

\section{Dialogue Game Framework}

\subsection{Model}

We consider recurrent dialogue patterns as conventional devices used by dialogue participants to coordinate their communicative actions in dialogue seen

\footnotetext{
${ }^{1}$ Action-oriented game types are: Offer, Request, Suggestion. Information-seeking game types are: OpenInterrogation, ChoiceGame, VerificationGame, NegativeVerificationGame, YNInterrogationGame.
} 
as a shared, dynamic and opportunistic activity. From our perspective, conventions can fruitfully be captured by a dialogue game structure to improve the conventional communicative capabilities of agents interacting with a human. A dialogue game is a conventional bounded joint activity between an initiator and a partner. Rules of the dialogue game specify the expected moves ${ }^{2}$ for each DP. Participants are expected to play their roles by making moves according to the current stage of the game. This activity is temporarily activated during the dialogue for a specific goal (e.g., information-seeking, action-seeking).

A detailed account of our formalisation of the dialogue game structure can be found in previous work[16]. We provide here an overview of our model, sufficient for the purposes of this article. A dialogue game is defined by a type and a subject. For instance, "Request(addKeyword(heart))" represents a dialogue game of type "Request" with subject "addKeyword(heart)" (which is the formalisation of the action "add the keyword 'heart' to the query", further described in Section 6.1.1). A dialogue game is a bilateral and shared structure which defines for the initiator of the game and for the partner (see for instance table 1):

- the entry conditions, which must hold before entering the game;

- the exit conditions, which define the success or failure status of the game;

- the rules, which define the expectations/obligations of dialogue participants in the game;

- and the effects of dialogue moves in the context of this game.

These aspects of dialogue games are modelled through the notions of social commitment and commitment store, inspired by dialectical systems[33, 45]. Social commitments are commitments that bind a speaker to a community[46]. They are public (unlike mental states such as belief, desire, intention), and are stored in a commitment store. Our formalisation classically distinguishes propositional commitments from action commitments. Propositional commitments concern those that do not deal with future actions such as when $x$ says "Charlie is well-known.". On the other hand, action commitments concern those dealing with future actions such as "I will make you a drawing tonight.". Thus, they represent actions that the agent is committed to perform in the future. Besides, action commitments may be either dialogical (they are valid in the context of a game) or extra-dialogical (their validity is not subject to a game). Social commitments are stored in a certain state. States can be distinguished between the default state (inactive, Ina), the state in which the commitment is active (created, Crt) and various states of inactivity subsequent to a period of activation (cancelled $(\mathbf{C n l})$, fulfilled $(\mathbf{F u l})$ and failed $(\mathbf{F a l}))$. The state of social commitments evolves through operations applied on the commitment

\footnotetext{
${ }^{2}$ Moves correspond to a context-change approach to dialogue acts[42, 44]. They take the form: $f(s, c)$ where $f$ is the communicative function, $s$ the speaker that produces this act and $c$ is the semantic content.
} 
store (e.g., creation, cancellation, satisfaction). Commitments are formalised as 4-arity predicates: $\mathrm{C}(x, y, c, s)$ (meaning that commitment " $x$ is committed towards $y$ about content $c$ " is in state $s$ ).

The content of action commitments can be combined in several ways including (but not limited to) the alternative $(\alpha \mid \beta)$ and the conditional statement $(\alpha \Rightarrow \beta)$ ( $\beta$ will occur if $\alpha$ does). Action commitments in our model make it possible to represent two high-level kinds of commitments: expectations/obligations and production rules. Expectations/obligations are represented with first-order commitments. For instance, when enclosed in a dialogical action commitment contracted by $y$, the content "acceptRequest $(y, \alpha) \mid \operatorname{declineRequest}(y, \alpha)$ " means that dialogue participant $y$ is expected to produce either an "acceptRequest" move, or a "declineRequest" move about action $\alpha$. Production rules encode conventional sequences of dialogue moves. They are represented with second-order commitments. For example, when enclosed in a dialogical action commitment contracted by $y$, the production rule "request $(x, \alpha) \Rightarrow \mathrm{C}_{\mathrm{g}}(y$,acceptRequest $(y, \alpha)$ declineRequest $(y, \alpha)$, Crt)" 3 specifies that the occurrence of the dialogue move "request $(x, \alpha)$ " commits dialogue participant $y$ to the production of an acceptance or a declination (by the activation of a first-order commitment).

All in all, the commitment store represents public commitments contracted by DPs at a given time of the interaction. These commitments represent positions about propositions, promises on the (non-)fulfilment of actions such as dialogue moves, and production rules encoding conventional sequences of dialogue moves. Furthermore, the commitment store contains a representation of dialogue games that are being suggested, currently being played, and closed.

\subsection{Example: the Request Game}

The definition of the "Request" dialogue game is presented in table 1. This game allows the initiator to request the partner to perform an action. Entry conditions specify that the partner must not be already committed on the requested action $\alpha$, i.e. either on its occurrence or its non-occurrence. The success conditions are the same for the two participants: the game is a success once the partner is committed to perform action $\alpha$. Similarly, the failure conditions state that the game is a failure if the attempt to commit the partner on $\alpha$ fails (i.e. the partner refuses to contract an active commitment about $\alpha$ ). Rules specify that the initiator of the game is committed to play a "request" dialogue move, and that the partner is committed to play an "acceptRequest" or a "declineRequest" move if $x$ plays a "request" move. Finally, effects precise that, in the context of this game, playing an "acceptRequest" or a "declineRequest" move for the partner commits him to perform $\alpha$, or to the failure of the committing attempt about $\alpha$.

Figure 1 illustrates the evolution of social commitments contracted by the dialogue participants in the context of a "Request" dialogue game. In particular, it highlights the application of production rules, and shows how social commit-

\footnotetext{
${ }^{3} g$ refers to the context of this dialogical commitment.
} 
Table 1: The Request Dialogue Game (explained in Section 4.2).

\begin{tabular}{|l|l|l|}
\hline$g=$ Request $(\alpha)$ & Initiator $(x)$ & Partner $(y)$ \\
\hline \hline Entry & & $\mathrm{C}(y, \alpha$, Ina $)$ and $\mathrm{C}(y, \neg \alpha$, Ina $)$ \\
Success & $\mathrm{C}(y, \alpha, \mathbf{C r t})$ & $\mathrm{C}(y, \alpha$, Crt $)$ \\
Failure & $\mathrm{C}(y, \alpha$, Fal $)$ & $\mathrm{C}(y, \alpha$, Fal $)$ \\
\hline Rules & request $(x, \alpha)$ & $\begin{array}{l}\text { request }(x, \alpha) \Rightarrow \mathrm{C}_{\mathrm{g}}(y, \text { acceptRequest }(y, \alpha) \mid \\
\text { declineRequest }(y, \alpha), \text { Crt })\end{array}$ \\
\hline Effects & & $\begin{array}{l}\text { acceptRequest }(y, \alpha) \Rightarrow \mathrm{C}(y, \alpha, \text { Crt }), \\
\text { declineRequest }(y, \alpha) \Rightarrow \mathrm{C}(y, \alpha, \text { Fal })\end{array}$ \\
\hline
\end{tabular}

ments generate conventional motivations for the production of certain moves. For the sake of readability, this example only involves a single dialogue game. Figure 1 presents partial views of the commitment store at three different times $\left(t_{1}, t_{2}\right.$ and $\left.t_{3}\right)$. It shows the content of the contracted action commitments that are currently active for the initiator of the game $(x)$ and for the partner $(y)$. At $t_{1}$, the dialogue game has been established and its rules are activated. The only expected dialogue move is a "request" move from $x$. Then, $x$ plays this move leading to $t_{2}$. This occurrence satisfies the commitment of $x$, and develops the production rule on which $y$ was engaged. At $t_{2}$, expected moves are either an "acceptRequest" move, or a "declineRequest" move from $y$. Then, $y$ produces the expected "acceptRequest" move leading to $t_{3}$. This occurrence satisfies the previous alternative as well as one of the effects of the game. It also commits $y$ to perform the action $\alpha$. Success conditions of the game are now met. Dialogue participants can now close this game.

Table 2 presents an example of dialogue involving this dialogue game (formal descriptions of other involved dialogue games such as "ChoiceGame" can be found in previous work[16]). Utterances 11 and 12 constitute the body of a "Request" dialogue game about the subject "addKeyword(heart)", initiated by interlocutor $y$ with partner $x$. This adjacency pair emerges from the conformance to the rules of the dialogue game. Interlocutor $y$ plays a "request" move in utterance 11 in the context of the "Request" dialogue game. The partner $x$ is thus committed to play either an acceptance or a declination (as shown in the "Expected moves" column). Interlocutor $x$ chooses to play an "acceptRequest" move in utterance 12, thus committing himself to perform the action "addKeyword(heart)". Hence, success conditions of this game are reached.

\subsection{The Contextualisation Game}

In addition to dialogue games, our model includes communication games dedicated to more general interaction processes (e.g., mutual understanding or turntaking). These games are simpler in the sense that they are always activated. Hence, their structure comes down to a set of rules expressed as dialogical action commitments. 
Table 2: Excerpt from a query building dialogue on a medical search engine between two interlocutors $(x$ and $y$ ) involving several dialogue games (see Sections 4.2 and 5.3). The "Move" column shows the communicative function being played. The "Game" column presents the game in which the move occurs. The "Expected moves" column describes the communicative functions of the moves that are expected according to the commitment store (" $<$ " is the priority relation, "l" represents an alternative).

\begin{tabular}{|c|c|c|c|c|}
\hline & Move & Game & Utterance & Expected moves \\
\hline 1 & prop.in & Contextualisat & $\begin{array}{l}\text { : May I suggest some } \\
\text { keywords? }\end{array}$ & $y: \operatorname{acc}$ in $^{*} \mid$ ref.in \\
\hline 2 & acc.in ${ }^{*}$ & - & $y:$ Yes. & $x$ : choiceQuestion $^{+}$ \\
\hline 3 & choiceQuestic & nChoiceGame & $\begin{array}{l}x: \text { Which keyword } \\
\text { would you choose } \\
\text { among: } \\
\text { 'myocardium', 'infarc- } \\
\text { tion', 'angiocardiogra- } \\
\text { phy'? }\end{array}$ & $y:$ answer $\bullet^{\bullet}$ ignore \\
\hline 4 & answer $\bullet$ & - & $\begin{array}{l}y: \text { Well, not 'infarc- } \\
\text { tion'... }\end{array}$ & $y$ : answer ${ }^{\bullet}$ ignore \\
\hline 5 & answer & - & $\begin{array}{l}\text {...not not 'angiocardio- } \\
\text { graphy'. }\end{array}$ & $y:$ answer | ignore \\
\hline 6 & prop.in & Contextualisat & $\begin{array}{l}\text { Can I ask you some- } \\
\text { thing? }\end{array}$ & $x$ : acc.in ${ }^{\dagger} \mid$ ref.in $<y$ : answer | ignore \\
\hline 7 & acc.in $^{\dagger}$ & - & $x:$ Sure! & $y$ : setQuestion $^{\circ}<y$ : answer $\mid$ ignore \\
\hline 8 & setQuestion $^{\circ}$ & OpenInterroga & $\begin{array}{l}\text { og: What is the defini- } \\
\text { tion of the term 'my- } \\
\text { ocardium'? }\end{array}$ & $x$ : answer $^{\star} \mid$ ignore $<y$ : answer $\mid$ ignore \\
\hline 9 & answer* & - & $\begin{array}{l}x \text { : A definition of 'my- } \\
\text { ocardium' is: 'The } \\
\text { muscle tissue of the } \\
\text { heart. It is composed } \\
\text { of [...]' }\end{array}$ & $y:$ answer ${ }^{\bullet}$ ignore \\
\hline 10 & answer $\bullet$ & ChoiceGame & $\begin{array}{l}y \text { : Then, I would } \\
\text { choose } \\
\text { 'heart'! }\end{array}$ & \\
\hline 11 & request & Request & $\begin{array}{l}\text { Please, add this } \\
\text { term to the query. }\end{array}$ & $\mathrm{x}$ : acceptRequest ${ }^{\diamond} \mid$ declineRequest \\
\hline 12 & acceptReques & $t \underline{-}$ & $x:$ All right! & \\
\hline
\end{tabular}




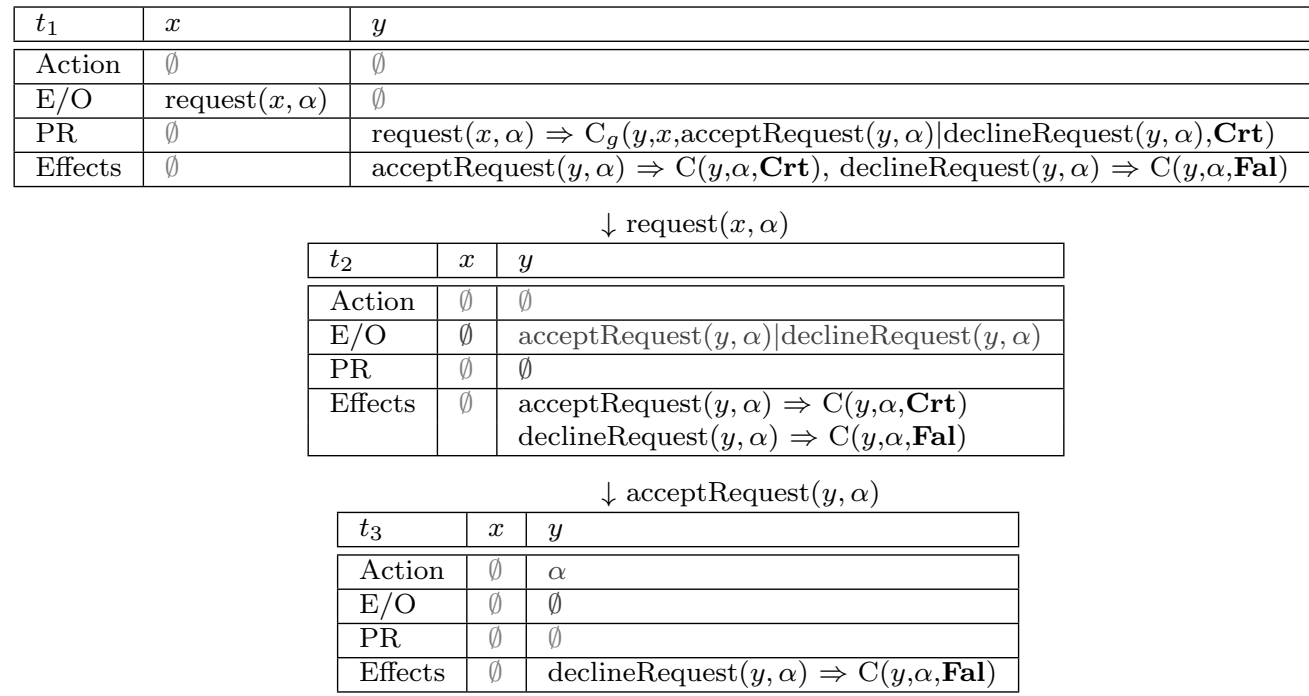

Figure 1: Example of the evolution of a partial view of the commitment store during a "Request" dialogue game. $x$ is the initiator, $y$ is the partner. $\mathrm{g}=\operatorname{Request}(\alpha) . \quad \alpha=\operatorname{addKeyword}\left(\right.$ heart). $\quad t_{1}<t_{2}<t_{3}$. $\mathrm{E} / \mathrm{O}=$ Expectations/Obligations, $\mathrm{PR}=$ Production rules.

As already mentioned, dialogue is a joint and opportunistic activity[10] in which DPs coordinate their contributions to co-construct and co-control the dialogue. Dialogue games are managed by DPs in a process that presents three typical phases: the entry, the body and the exit of the joint activity. Establishment and closing of dialogue games have been described by a negotiation metaphor[47]. Our model includes the communication game of contextualisation which implements a version of this process that may involve two proposition phases (one for the entry and one for the exit). Dialogue example in table 2 presents two explicit entry phases: one for a choice dialogue game initiated by $x$ (utterances 1-2) and another for an open interrogation dialogue game initiated by $y$ (utterances $6-7$ ). Entry phases follow a common structure showing a proposition to enter the game (via a "prop.in" move) followed by an acceptance (via an "acc.in" move). Structure of an explicit exit phase is similar to the one of an entry phase. Exit phases can be realised implicitly via the reaching of success or failure conditions of the dialogue game.

\section{Dialogue Games and Dialogue Management}

\subsection{Interpretative and Generative Aspects of our Model}

H-M dialogue models inspired by dialectical ones present a strong interest for modelling $\mathrm{H}-\mathrm{M}$ interaction[34]. Their main advantage lies in their normative 
nature in that they constrain the communicative behaviour of the DPs. From a computational perspective, these models have two important interests for an interactive agent. They present an interpretative interest that makes it possible to determine the legality of a dialogue move produced by a DP. Therefore, an agent following a dialogue game based model can adapt its reaction according to whether a dialogue move is legal or not. Besides, this kind of model also presents a generative interest: it makes it possible to assist the system for the production of a response by limiting its attention to the legal moves that are expected from the agent.

The normative aspect of our model can be found in the idea of dialogue games as structures capturing social commitments (i.e. that encode conventions of human interaction). In the course of interaction, DPs gradually contract social commitments that not only constrain the communicative behaviour of the system, but that of the human participant as well.

From an interpretative point of view, our model considers the legality of a dialogue move from different perspectives. A dialogue move can be forbidden or expected depending on contracted social commitments. Among a list of expected dialogue moves, a particular one can have priority based on the partial ordering of social commitments (legality and priority are exemplified in Section 5.3). The interpretative role of a dialogue manager based on dialogue games is to classify a dialogue move according to these criteria, and react accordingly. This is exemplified by the generic algorithm 5.1. The dialogue move that just occurs is first assessed against the contracted social commitments (line 1). Then, it is processed according to its legality status: a legal dialogue move (i.e. an allowed, expected and priority move) leads to a standard update of the dialogue context (line 6). Other cases (i.e. forbidden, non-priority or unexpected move) involve a case-specific dialogue policy (lines 3, 8, 11). From our perspective, these policies depend on the interactive agent that is being designed. For instance, forbidden dialogue moves can simply be prohibited in debate situations[48]. Notably, our model organizes the interpretative communicative behaviour of an agent for known games in a generic fashion. In other words, a move that cannot be integrated by the system as being a participation in a known game would be treated as an "unexpected" move (as would be the case, e.g., if the human participant plays a move from a dialogue game unknown to the agent). Dealing with such moves has to be done on an application-dependent basis. However, one advantage of our model is to make it possible to detect unexpected dialogue move as being "illegal". Then, several strategies can be adopted by the system to ensure robustness and to overcome such a situation. For instance, one strategy can be to display to the user the illegality of the move and the inability of the system to deal with it (e.g., "I cannot understand what you mean. Please, try something else."). Another one can be to call a "fallback strategy" to avoid this unexpected move while maintaining user engagement by initiating a fallback dialogue game[49]. Another one can be to switch from the interactive agent to a human operator that takes control of the agent to continue the interaction.

From a generative point of view, our model makes it possible to compute the conventionally expected dialogue moves from the system by examining its 


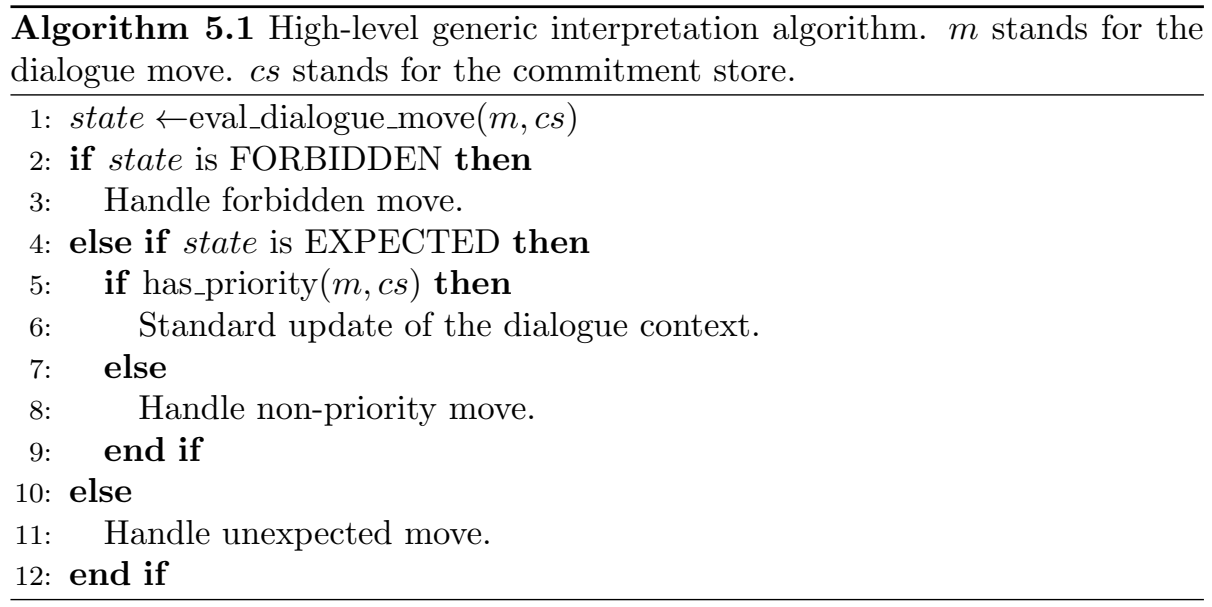

contracted dialogical commitments. It provides conventional reasons to the production of dialogue moves by taking into account the state of the commitment store as well as the activated dialogue games. These elements should be considered during the deliberative process of the interactive agent.

\subsection{Implementation: Dogma Module}

DoGma (for "DialOgue Game MAnager") is a dialogue game-based normative module that manages conventional interaction in a two-interlocutor dialogue. It is designed as a part of a dialogue manager of an interaction system. Dogma adopts the information state-based approach to dialogue management[44]. It is in charge of the update and the exploitation of the commitment store located in the information state of the dialogue manager.

Figure 2 presents the architecture of DoGma. The system is centred around the information state, classically divided into two parts: the public part that includes the commitment store, and the private part. The commitment store is the main component of DoGMA. It consists of a partially ordered set of social commitments contracted by the speakers. To be more specific, it contains for each DP the public commitments in terms of propositions (as extra-dialogical commitments) and actions (as extra-dialogical and dialogical commitments). Besides, it contains a representation of dialogue games that are being suggested, currently being played, and closed. The commitment manager has a read/write access to the commitment store. Its role is to keep the commitment store up to date after the occurrence of events such as dialogue moves. Dogma provides two components with a read access to the commitment store: (i) the referee that evaluates the legality of a dialogue move (cf. algorithm 5.1), and (ii) the conventional behaviour manager that identifies the conventionally expected dialogue moves from each DP. The referee and commitment manager components are solicited during the interpretative phase of the dialogue manager control algorithm. The conventional behaviour manager is mainly called during the 


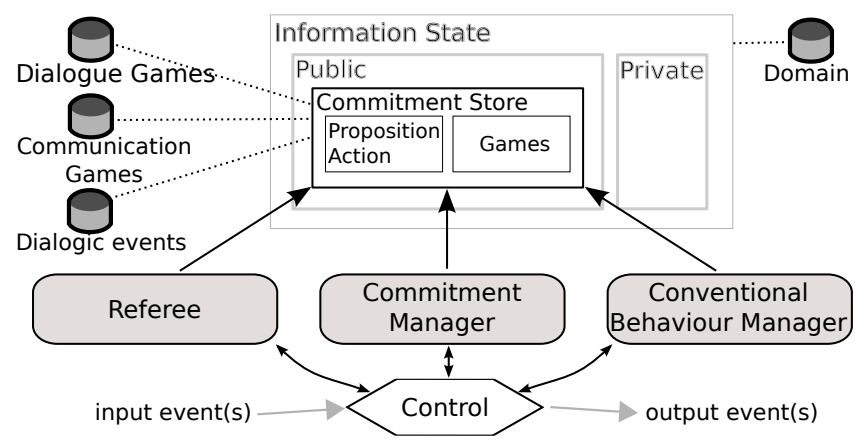

Figure 2: Dogma Architecture

generative phase of the control algorithm.

Dogma is configured by three main resources: (i) the dialogic events library, (ii) the communication games library, and the (iii) dialogue games library. These resources define the dialogue moves and games that are available during the interaction. Last but not least, the domain library brings the domain-dependent elements of the application, namely: (i) the semantics of the dialogue move content, and (ii) a specification of the allowed combinations between dialogue games.

We have developed Dogma using the Scala[50] programming language ${ }^{4}$. The source code is freely available under the GPLv3 licence ${ }^{5}$, and can currently be found at https://labanquise.insa-rouen.fr/projects/dogma/.

\subsection{Example of Usage of Dogma}

Table 2 presents an excerpt of a query building dialogue on a medical search engine between two DPs. It shows how a dialogue manager can take advantage of Dogma to manage the conventional part of the interaction. From utterance 1 to 10 is shown the playing of a choice dialogue game initiated by $x$ (in utt. 1-2) that is interrupted by an open interrogation game initiated by $y$ (in utt. 69 ), and followed by a request game previously described in section 4.2. First, this example shows that the body of a dialogue game is not restricted to a pair of moves but can span several ones (e.g., utt. 3, 4, 5 and 10). Next, DoGmA allows the combination of dialogue games such as embedding (cf. utt. 69). A consequence of such a combination happens on the priority of social commitments representing move expectations: moves expected in the embedded game take precedence over those of the parent game. For instance, an answer to the open interrogation game has priority over moves of the choice game after utt. 8, due to conversational precedence (the same occurs after utt. 6 and 7). Eventually, this example exposes the expected moves derived from the commitment store in the last column of the table. These expected moves are

\footnotetext{
${ }^{4}$ See http://www.scala-lang.org/

${ }^{5}$ See http://www.gnu.org/copyleft/gpl.html
} 
computed by the conventional behaviour manager which provides legal moves that are conventionally expected from DPs given the current dialogue state. As such, they represent both conventional expectations from the other DP and conventional reasons to the production of given moves. For example, after utt. 6, interlocutor $y$ conventionally expects an acceptation or declination about the establishment of the open interrogation game, and still has conventional motivations to respond to the choice question asked by $x$ in utt. 3 .

\section{Evaluation}

Our goal is to evaluate the ability of our system to generate short fragments of interaction that are meaningful to humans (while being combinable by a dialogue planner). We adopted a validation process as a test "a la Turing". It consists in comparing a set of $\mathrm{H}-\mathrm{H}$ interaction patterns observed in our corpus to a set of interaction patterns automatically generated by our system. This variant involves experts whose task is to analyse a sample of interaction patterns (mixing real ones and generated ones) in order to determine if the two sets can easily be distinguished. This experiment leads us to the creation of four databases of dialogue patterns: (i) three databases of automatically generated interaction patterns by three different processes, and (ii) a database of interaction patterns from $\mathrm{H}-\mathrm{H}$ interactions.

\subsection{Databases of Interaction Patterns}

\subsubsection{Databases of Generated Patterns}

Semantic Representation We first manually established a semantic database of questions, propositions and actions occurring in the dialogue patterns of the study corpus. This step completes a work initiated in [15]. Our formalisation is a reduced semantic representation with a domain-dependent level of granularity similar to the one proposed by [51]. It is based on predicate logic without quantification. The use of such a simple semantic representation for practical dialogue systems is well-argued in $[8,9]$. Our representation of questions classically distinguishes yes/no questions, choice questions and wh-questions. Each element of the semantic database is given a standard translation in French language. Table 3 presents some constituents of the semantic database. All in all, our semantic database contains 1,507 instances of actions, 1,121 instances of propositions and 2,086 instances of questions.

Automatic Pattern Generation We considered three automatic pattern generation algorithms: a random mode and two Dogma-based modes. These three modes produce interaction patterns as sequences of dialogue moves.

The random mode generates a pattern by randomly selecting for each move: a locutor (among two), a communicative function and a consistent semantic content. The communicative function is picked from $19 \mathrm{DIT}++$ general-purpose 
Table 3: Examples from the semantic database.

\begin{tabular}{|l|l|l|}
\hline Example & Type & Description \\
\hline \hline addKeyword(food) & Action & $\begin{array}{l}\text { Add keyword "food" to the } \\
\text { query. }\end{array}$ \\
\hline ?X.definition(eczema, $X)$ & Wh-question & $\begin{array}{l}\text { What is the definition of } \\
\text { "eczema" ? }\end{array}$ \\
\hline$\{$ ?patient,?student,?doctor $\}$ & Y/N question & $\begin{array}{l}\text { Is there a subheading applicable } \\
\text { to "headach" in the terminol- } \\
\text { ogy? }\end{array}$ \\
\hline hyponym(heart, myocardium) & Proposition & $\begin{array}{l}\text { Are you a patient, a student or } \\
\text { a doctor? }\end{array}$ \\
\hline
\end{tabular}

functions that occurred in interaction patterns of the study corpus. Pattern lengths are uniformly distributed between 2 and 5 .

The first Dogma mode generates patterns based on a simple deliberative process that takes into account the information state and that exploits the semantic database. First, a dialogue game type and a compatible goal are chosen. Types are picked from a set that includes 3 action-oriented game types and 5 information-seeking-oriented game types. The probability to select a type corresponds to the distribution of types observed in the study corpus. Next, one of the DP is designated initiator of the game whereas the other is designated partner. The initiator plays an explicit proposition to establish the dialogue game. Then, the automatic generation starts. It takes advantage of the conventional behaviour manager of DoGMA to compute a time-ordered set of expected dialogue moves from the DPs. The algorithm that selects the next dialogue move takes into account: (i) the priority of an expected dialogue move (the more recent, the greater the priority is), and (ii) the interaction history (DPs cannot repeat an already produced dialogue move). The generation stops when no more dialogue moves are expected.

The second Dogma mode simulates the implicit establishment of the game. It thus works similarly to the first one but only keeps the body of the pattern (entry and exit phases are discarded).

Natural Language Generation Dialogue patterns are given a natural language representation through a simple template-based generation mechanism. For each dialogue move of a pattern, a template is selected and then applied. Several templates are available for a given dialogue move. Templates are randomly chosen. Generation templates have been manually crafted by extracting cue-phrases specific to communicative functions at the surface level of utterances of our study corpus. These templates obviously are language- and domaindependent. 


\subsubsection{Database of $\mathrm{H}-\mathrm{H}$ Patterns}

The database of $\mathrm{H}-\mathrm{H}$ interaction patterns was established by extracting all recurrent dialogue patterns from our reference corpus (the process was similar to the one executed on the study corpus, cf. section 3). Interaction patterns were post-processed on their surface level to obtain a normalised form. This process consisted in the removal of transcription signs (e.g., pause indicator), in the normalisation of punctuation and in the correction of spelling mistakes. In short, we extracted $44 \mathrm{H}-\mathrm{H}$ dialogue patterns which mainly are adjacency pairs. An example of such a pattern from our corpus is: "- What I propose is to remove the subheading from the query. - Alright!" (translated from French to English).

\subsection{Experiment}

Our experiment consists in the qualitative assessment of the interaction patterns obtained by the previously described means. Evaluation database contains 176 patterns (44 per mode). We considered two dimensions to carry out our comparative study: (i) coherence (a pattern is said to be coherent if it does not contain a dialogue contribution which presence is not easily explained by taking into account the previous ones [47]), and (ii) naturalness (a pattern is "natural" if it seems to have been produced by a H-H interaction).

We have performed the evaluation on the web, in French. Participants were confronted to a random selection of patterns to avoid any effect on the results. For each pattern, we asked the participants to answer two questions (one per dimension) using a Likert scale of 4 points (e.g., "Coherent", "Rather coherent", "Rather incoherent", "Incoherent"). Participants were recruited via French mailing lists. 89 French-native individuals have participated in this evaluation (26 females, 63 males). $75 \%$ of them are between 20 and 39 years old (min=19, $\max =64)$.

\subsection{Results}

We collected 2,960 evaluations of pattern uniformly distributed over the 4 database types.

\subsubsection{Perceived "Coherence" of the Patterns}

Cumulative coherence results for each group are presented in figure 3 .

Two different clusters in terms of coherence can clearly be distinguished. The first one unites the set of $\mathrm{H}-\mathrm{H}$ patterns and the two DoGmA-generated sets of patterns. It is characterised by a common value of mode and median which is "Coherent", the highest level of coherence. The other cluster consists of the set of random-generated patterns (mode=median= "Incoherent", the lowest level of coherence). Results for the random mode shows that humans are able to clearly assess coherence of short dialogue patterns. 


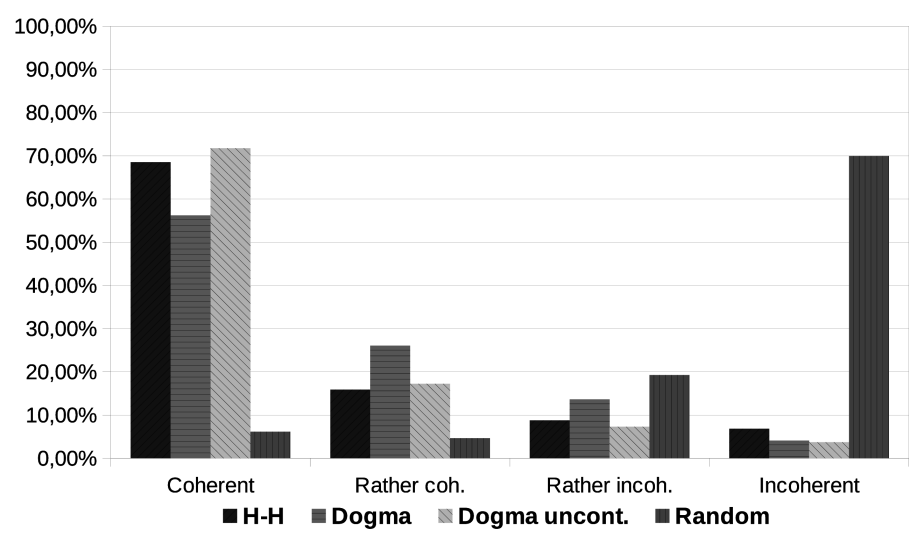

Figure 3: Distribution of coherence results per pattern groups. "Dogma impl." stands for the second Dogma-based generation mode.

We applied a statistical test to quantify a distance between the empirical coherence distribution of the H-H group and the other ones (DogmA, Dogma impl. and random). Due to the ordinal nature of our data and the large size of our samples, we selected the two-sample Kolmogorov-Smirnov test with a significance level $\alpha=0.05$. It turns out that the empirical coherence distributions of H-H and "Dogma impl." groups can be said to come from the same distribution $(D=0.0459$, p-value $=0.412)$. However, this strong hypothesis is rejected for the Dogma group $\left(D=0.123\right.$, p-value $\left.=2.471 \times 10^{-05}\right)$ and for the random one $\left(D=0.736, \mathrm{p}\right.$-value $\left.=2.849 \times 10^{-173}\right)$. This test shows that there is no significant statistical difference between the observed empirical coherence distributions of the H-H group and the "DoGma impl." one.

Figure 4 takes a closer look at coherence results by presenting cumulative coherence results for each pattern type.

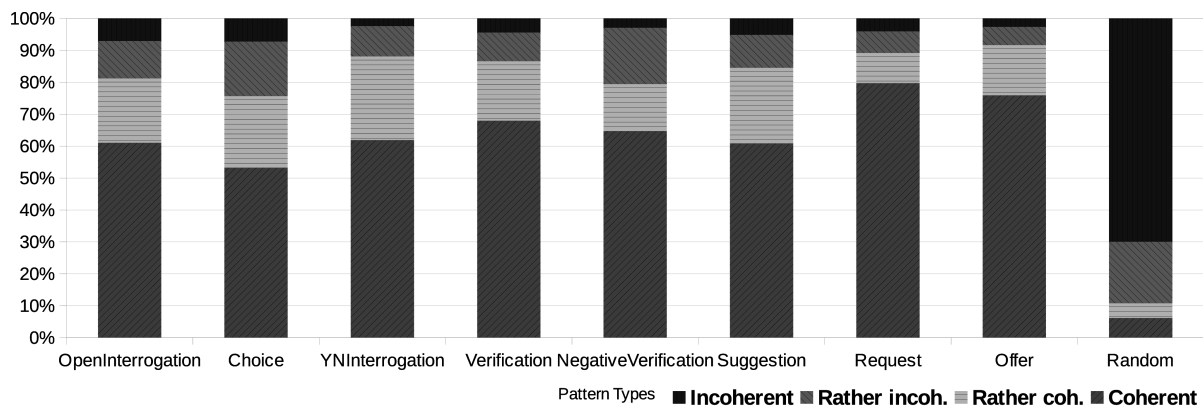

Figure 4: Distribution of coherence results per pattern types (8 dialogue games and the random mode).

Two groups of pattern types can be observed. The first one consists of the 
random-generated patterns which have been unsurprisingly rated as incoherent ( mode $=$ median $=$ "incoherent").

The second one consists of the 8 dialogue games of our library. It is characterised by a common value of mode and median in terms of coherence which takes the maximum value "Coherent". This indicates that the perceived coherence of the patterns is independent of the pattern types to a large extent. However, a certain variability between the pattern types can be observed. Indeed, it ranges from approx. $75 \%$ of pattern evaluations that have been rated as "coherent" or "rather coherent" for the "Choice" dialogue game to around $91 \%$ for the "Offer" dialogue game. From our perspective, two main factors can explain this variability. The first one is that information-seeking games (such as the "OpenInterrogation" and "Choice" games) are more elaborate than actionoriented games (such as the "Request" and "Offer" games) in the sense that they involve a wide variety of responses going beyond simple acceptance or refusal. Then, information-seeking games may suffer from the NLG process which may impact both "coherence" and "naturalness" (further developed in Section 6.3.2).

These results can be further developed by looking at the perceived coherence both by dialogue game types and by generation mode ( $\mathrm{H}-\mathrm{H}$, Dogma, Dogma impl.). It turns out that similar results can be observed for both the $\mathrm{H}-\mathrm{H}$ and the Dogma impl. modes: for each generation mode and for each dialogue game type, the mode and median in terms of coherence are at the "Coherent" value. Results for the Dogma mode are slightly different. Mode and median are also to the "Coherent" value for every pattern types at the exception of the "YNinterrogation" game and the "Choice" game where the median is at the "Rather coherent" value.

All in all, our study shows that humans are able to clearly assess coherence of short dialogue patterns. Patterns generated randomly have been distinctly qualified as being incoherent. Patterns generated by the Dogma impl. mode maintain similar results than the $\mathrm{H}-\mathrm{H}$ patterns in terms of perceived coherence both globally and by pattern types. Patterns generated by the DoGma mode obtains slightly inferior results. While being perceived coherent, patterns generated by this mode seems to suffer from the explicit version of the game establishment process.

\subsubsection{Perceived "Naturalness" of the Patterns}

Participants were asked to assess the "naturalness" of the patterns in the same fashion than for the coherence, i.e. using a Likert scale of 4 points ("Humanlike", "Rather human-like", "Rather non-human-like", "Non-human-like"). There is a link between the "coherence" and the "naturalness" results. Indeed, it exists a significant linear correlation between those results for the evaluated patterns $\left(r=0.72\right.$, t-test p-value $\left.=2.2 \times 10^{-16}\right)$, computed with Pearson's productmoment correlation. This is not surprising: it is indeed reasonable to expect that a pattern perceived as "human-like" is also perceived as being "coherent". However, these two aspects should not be confused as this is shown by the analysis presented below. 
Cumulative "naturalness" results for each group are presented in figure 5. An analysis of these results reveals three different clusters. The first one consists of the set of H-H patterns (mode=median= "Human-like", the highest level). The second one is made of the sets of the two DogmA modes (mode= "Human-like", median= "Rather human-like"). The third one contains the random mode which has been perceived "Non-human-like" (the lowest level). DoGMA-generated patterns are distinguishable from $\mathrm{H}-\mathrm{H}$ patterns while being mainly seen as humanlike. One obvious reason is that we use a simplistic template-based NLG system. On the other hand, random patterns are adequately perceived as being non-human-like.

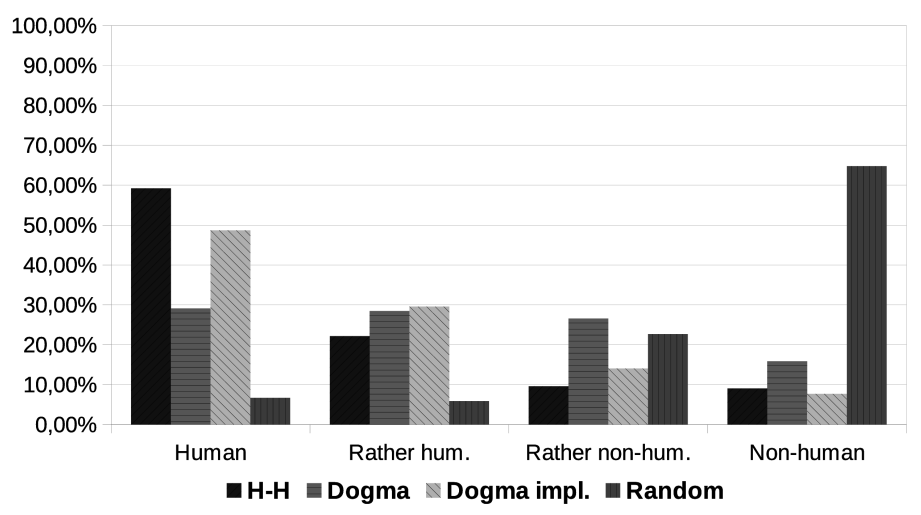

Figure 5: Distribution of naturalness results per pattern groups. "DoGmA impl." stands for the second DoGmA-based generation mode.

Tables 4 and 5 present the mode and median results for each dialogue game type and for each generation mode. $\mathrm{H}-\mathrm{H}$ patterns have been mainly perceived as "Human-like", which they are, independently of the dialogue game types ( mode $=$ median $=$ "Human-like").

Results for the two Dogma-based modes are mixed. A distinction can be observed between those two modes. DoGmA impl. mode obtains similar modes and medians than the $\mathrm{H}-\mathrm{H}$ mode for half of the pattern types (namely, the Choice, Verification, NegativeVerification and Request patterns). It obtains slightly inferior results for the other half, the median being "rather human-like". DoGmA mode obtains similar or slightly inferior results compared to the DoGmA impl. mode on 5 pattern types (namely, the Verification, NegativeVerification, Suggestion, Request and Offer patterns). Notably, a clear difference appears on three information-seeking pattern types (the OpenInterrogation, Choice and YNInterrogation pattern types). They are perceived as "(rather) human-like" when generated by the DoGmA impl. mode whereas they are perceived as "rather non-human-like" when generated by the DoGmA mode. The only difference between these two modes being the establishment of the games (explicit for the Dogma mode, implicit for the other), this reveals that the establishment process has an impact on the "naturalness" of the generated pattern (while seeming 
Table 4: Mode and median "naturalness" results for information-seeking game types and for each generation mode. $\mathrm{H}=$ "Human-like", $\mathrm{RH}=$ "Rather humanlike", $\mathrm{RNH}=$ "Rather non-human-like".

\begin{tabular}{|c|c|c|c|c|c|c|c|c|}
\hline \multirow[t]{2}{*}{ Gen. mode } & \multicolumn{2}{|c|}{ OpenInterrog. } & \multicolumn{2}{|c|}{ Choice } & \multicolumn{2}{|c|}{ YNInterrog. } & \multicolumn{2}{|c|}{ Verification } \\
\hline & Mode & Median & Mode & Median & Mode & Median & Mode & Median \\
\hline $\mathrm{H}-\mathrm{H}$ & \multicolumn{2}{|c|}{$\mathrm{H}$} & & $\mathrm{H}$ & \multicolumn{2}{|c|}{$\mathrm{H}$} & & $\mathrm{H}$ \\
\hline DOGMA impl. & $\mathrm{H}$ & $R H$ & & $\mathrm{H}$ & $\mathrm{H}$ & $R H$ & & $\mathrm{H}$ \\
\hline DOGMA & \multicolumn{2}{|c|}{ RNH } & & NH & \multicolumn{2}{|c|}{ RNH } & \multicolumn{2}{|c|}{$R H$} \\
\hline
\end{tabular}

Table 5: Mode and median "naturalness" results for action-oriented game types and for each generation mode. $\mathrm{H}=$ "Human-like", $\mathrm{RH}=$ "Rather human-like", $\mathrm{RNH}=$ "Rather non-human-like".

\begin{tabular}{|l|c|c||c|c||c|c|}
\hline \multirow{2}{*}{ Gen. mode } & \multicolumn{2}{|c||}{ Suggestion } & \multicolumn{2}{c||}{ Request } & \multicolumn{2}{c|}{ Offer } \\
\cline { 2 - 7 } & Mode & Median & Mode & Median & Mode & Median \\
\hline \hline H-H & \multicolumn{2}{|c|}{$\mathrm{H}$} & \multicolumn{2}{|c||}{ H } & \multicolumn{2}{|c|}{ H } \\
\hline DOGMA impl. & $\mathrm{H}$ & $R H$ & \multicolumn{2}{|c|}{$\mathrm{H}$} & $\mathrm{H}$ & $R H$ \\
\hline DoGMA & $\mathrm{H}$ & $R H$ & $\mathrm{H}$ & $R H$ & $\mathrm{H}$ & $R H$ \\
\hline
\end{tabular}

coherent). Besides, this mainly affects the information-seeking game category (3 games out of 5 ).

To conclude, this study shows that short interaction patterns generated via the two Dogma modes appear to a certain extent as coming from a $\mathrm{H}-\mathrm{H}$ interaction. However, they are distinguishable from real $\mathrm{H}-\mathrm{H}$ patterns. Two main factors can explain why the DoGMA-generated patterns appear less human-like. First, this can be explained by the use of a simplistic template-based NLG system. This introduces redundancy in the generated language that can be easily detected by a human. Then, a comparison between the two Dogma modes points out the explicit establishment of a dialogue game as being non-human-like for some information-seeking games. This indicates that the explicit establishment may be too cumbersome from a human perspective for some games, while being perceived as a coherent structure of language use.

\subsection{Discussion}

We have outlined interesting properties of our dialogue game-based model. First, we have presented its ability to model in a computational way empirically observed H-H dialogue patterns. Next, we have described how this model can be useful to manage the conventional communicative behaviour of an interacting agent, in particular by its discernment of legal moves from the current dialogue state. Notably, this model allows to manage dialogue in cases where a conventional response is sufficient, thus avoiding a complicated and difficult to set up plan-recognition process. Then, this framework includes mechanisms for the cocontrol of dialogue by both participant which could be useful in mixed-initiative 
interaction[4]. Last, we have shown that this model succeeds to produce a human level of coherence on both semantic and pragmatic levels on short dialogue patterns, contrary to the weak coherence reached by Orkin et al.[13]. Our model thus seems very promising to manage the low-level communicative behaviour of an interactive agent while being integrable to a high-level deliberative process (e.g., through dialogue plans[51], activation networks[52]).

Besides, our study has made it possible to identify two factors playing an important role to generate human-like interaction patterns. One is, unsurprisingly, the natural language generation process to turn a dialogue act into a natural language form. The other is the game establishment process of our model that should take either an explicit or an implicit form depending on the game type.

\section{Conclusions and Future Work}

In this work, we have seen how dialogue patterns can be fruitfully exploited to enrich high-level structures for dialogue management, namely, dialogue games seen as structures capturing commitments. These games are building blocks of conventional interactions that an agent could employ to interact with a human. We have pointed out computational advantages of these structures on both interpretative and generative levels of dialogue management. We presented DoGMA, an open-source module that can be used by an interactive agent to manage its conventional communicative behaviour in a two-interlocutor dialogue. We have shown that our library of empirically specified dialogue games can be exploited into Dogma to generate fragments of dialogue that are: (i) coherent from a human perspective at the semantic and pragmatic levels, and (ii) perceived mainly as human-like while being distinguishable from real $\mathrm{H}-\mathrm{H}$ interaction patterns.

Future work includes two interesting perspectives at the model level. One is the study of the implicitness of the dialogue game establishment process, that is not perceived as being "natural" in its explicit form for some games. Another is the design of a high-level deliberative model that takes into account dialogue games and the opportunistic nature of dialogue. Next, additional evaluation angles could be explored. One is the impact of the language choice on the coherence study. This would consist in conducting the experimentation in a language other than French (used in this article). Our dialogue model being at the dialogue act level, the main change required to perform this evaluation would be to adapt the NLG module. Another is conducting an experimentation confronting human patterns and automatically generated patterns in a Turing test, and thus forcing a choice of the more "human-like pattern". As explained in our discussion of the study, these new experimentations should rely on an improved NLG module to avoid redundancy in the natural language forms of automatically generated dialogue patterns. Eventually, rules of the dialogue games were predefined in this work by leveraging dialogue patterns extracted via a data-driven methodology. A promising perspective resides in the exploitation of unlabelled or labelled dialogue data to automatically discover dialogue patterns and to automatically learn dialogue game rules. 


\section{Acknowledgements}

This work was sponsored by the Upper Normandy region (France). It was partly supported by the NARECA project (ANR-13-CORD-0015). The authors would like to warmly thank the participants who took part in the experiments. The authors thank the anonymous reviewers for their valuable comments and suggestions.

\section{References}

[1] H. Lieberman, Autonomous interface agents, in Proceedings of the ACM SIGCHI Conference on Human factors in computing systems (ACM, 1997), pp. 67-74.

[2] J. Cassell, Nudge nudge wink wink: Elements of face-to-face conversation for embodied conversational agents, Embodied conversational agents (2000) $1-27$.

[3] W. R. Swartout, J. Gratch, R. W. Hill, Jr., E. H. Hovy, S. Marsella, J. Rickel and D. R. Traum, Toward virtual humans, AI Magazine 27(2) (2006) 96-108.

[4] G. Tecuci, M. Boicu and M. T. Cox, Seven aspects of mixed-initiative reasoning: An introduction to this special issue on mixed-initiative assistants, AI Magazine 28(2) (2007) p. 11.

[5] M. Schröder, E. Bevacqua, R. Cowie, F. Eyben, H. Gunes, D. Heylen, M. ter Maat, G. McKeown, S. Pammi, M. Pantic, C. Pelachaud, B. Schuller, E. de Sevin, M. F. Valstar and M. Wöllmer, Building autonomous sensitive artificial listeners, Transactions on Affective Computing 3(2) (2012) 165183.

[6] A. B. Youssef, M. Chollet, H. Jones, N. Sabouret, C. Pelachaud and M. Ochs, Towards a socially adaptive virtual agent, in International Conference on Intelligent Virtual Agents Springer2015, pp. 3-16.

[7] J. Mariani, S. Rosset, M. Garnier-Rizet and L. Devillers, Natural Interaction with Robots, Knowbots and Smartphones: Putting Spoken Dialog Systems Into Practice (Springer Science \& Business Media, 2014).

[8] K. Jokinen and M. McTear, Spoken Dialogue Systems (Morgan \& Claypool, 2010).

[9] F. Landragin, Man-Machine Dialogue. Design and Challenges. (WileyISTE, London, UK, 2013).

[10] H. Clark, Using language (Cambridge University Press, 1996). 
[11] J. Allwood, Obligations and options in dialogue, Think Quarterly 3 (1994) 9-18.

[12] J. Hulstijn, Dialogue games are recipes for joint action, in Workshop on the Semantics and Pragmatics of Dialogue 2000.

[13] J. Orkin and D. Roy, Automatic learning and generation of social behavior from collective human gameplay, in Proceedings of the 8th International Conference on Autonomous Agents and Multiagent Systems 1, (Richland, SC, 2009), pp. 385-392.

[14] J. Orkin, Collective artificial intelligence: simulated role-playing from crowdsourced data, $\mathrm{PhD}$ thesis, Massachusetts Institute of Technology2013.

[15] A. Loisel, G. Dubuisson Duplessis, N. Chaignaud, J.-P. Kotowicz and A. Pauchet, A conversational agent for information retrieval based on a study of human dialogues, in International Conference on Agent and Artificial Intelligence 2012 , pp. 312-317.

[16] G. Dubuisson Duplessis, N. Chaignaud, J.-P. Kotowicz, A. Pauchet and J.P. Pécuchet, Empirical Specification of Dialogue Games for an Interactive Agent, in Advances on Practical Applications of Agents and Multi-Agent Systems: 11th International Conference, PAAMS 2013, Salamanca, Spain, May 22-24, 2013. Proceedings, eds. Y. Demazeau, T. Ishida, J. M. Corchado and J. Bajo. (Springer Berlin Heidelberg, Berlin, Heidelberg, 2013), Berlin, Heidelberg, pp. 49-60.

[17] S. Darmoni, J. Leroy, F. Baudic, M. Douyere, J. Piot and B. Thirion, Cismef: a structured health resource guide, Methods of Information in Medicine 39 (2000) 30-35.

[18] Z. Ales, G. Dubuisson Duplessis, O. Serban and A. Pauchet, A methodology to design human-like embodied conversational agents, in $A A M A S$ Workshop, Human-Agent Interaction Design and Models2012.

[19] G. Dubuisson Duplessis, A. Pauchet, N. Chaignaud and J. P. Kotowicz, A conventional dialogue model based on empirically specified dialogue games, in 2015 IEEE 27th International Conference on Tools with Artificial Intelligence (ICTAI)2015, pp. 997-1004.

[20] W. Mann, Dialogue games: Conventions of human interaction, Argumentation (4) (1988) 511-532.

[21] B. Grosz and C. Sidner, Attention, intentions, and the structure of discourse, Computational Linguistics 12(3) (1986) 175-204.

[22] J. Allen, G. Ferguson, B. Miller, E. Ringger and T. Sikorski-Zollo, Dialogue systems: From theory to practice in TraINS-96, in Handbook of Natural Language Processing2000, pp. 347-376. 
[23] C. Rich and C. Sidner, COLLAGEN: a collaboration manager for software interface agents, User Modeling and User-Adapted Interaction 8(3) (1998) $315-350$.

[24] C. Rich and C. L. Sidner, Using collaborative discourse theory to partially automate dialogue tree authoring, in International Conference on Intelligent Virtual Agents2012, pp. 327-340.

[25] M. Sun, Y.-N. Chen and A. I. Rudnicky, An intelligent assistant for highlevel task understanding, in Proceedings of the 21st International Conference on Intelligent User Interfaces ACM2016, pp. 169-174.

[26] D. Griol and Z. Callejas, A proposal to develop domain and subtaskadaptive dialog management models, in Sixteenth Annual Conference of the International Speech Communication Association 2015.

[27] Y.-N. Chen, W. Y. Wang and A. I. Rudnicky, Unsupervised induction and filling of semantic slots for spoken dialogue systems using frame-semantic parsing, in Automatic Speech Recognition and Understanding (ASRU), 2013 IEEE Workshop on IEEE2013, pp. 120-125.

[28] D. Hakkani-Tur, Y.-C. Ju, G. Zweig and G. Tur, Clustering novel intents in a conversational interaction system with semantic parsing, in Sixteenth Annual Conference of the International Speech Communication Association 2015 .

[29] P. Cohen, Survey of the state of the art in human language technology (Cambridge University Press, New York, NY, USA, 1997) pp. 204-210.

[30] S. Pulman, The TRINDI project: Some preliminary themes, in Workshop on Language Technology 1998.

[31] L. Polanyi and R. Scha, A syntactic approach to discourse semantics, in International Conference on Computational Linguistics1984, pp. 413-419.

[32] J. A. Levin and J. A. Moore, Dialogue games: Metacommunication structures for natural language interaction, Cognitive Science 1(4) (1977) 395420.

[33] N. Maudet, Modéliser les conventions des interactions langagières: la contribution des jeux de dialogue, $\mathrm{PhD}$ thesis, Toulouse 32001.

[34] T. Yuan, D. Moore, C. Reed, A. Ravenscroft and N. Maudet, Informal logic dialogue games in human-computer dialogue, Knowledge Engineering Review 26(2) (2011) 159-174.

[35] G. Dubuisson Duplessis, Modèle de comportement communicatif conventionnel pour un agent en interaction avec des humains: approche par jeux de dialogue, $\mathrm{PhD}$ thesis, INSA de Rouen2014. 
[36] I. Lewin, A formal model of conversational game theory, in Workshop on the Semantics 83 Pragmantics of Dialogue 2000.

[37] M. Morge, F. Delecroix and J.-C. Routier, A virtual selling agent which is persuasive and adaptive, in Agreement Technologies, ed. S. Ossowski, Law, Governance and Technology Series 8 (Springer Netherlands, 2013) pp. $625-645$.

[38] J. Peltason and B. Wrede, Modeling human-robot interaction based on generic interaction patterns, in AAAI Fall Symposium: Dialog with Robots 2010 .

[39] J. Peltason, Modeling Human-Robot-Interaction based on generic Interaction Patterns, PhD thesis, Bielefeld University2014.

[40] A. Sauppé and B. Mutlu, Design patterns for exploring and prototyping human-robot interactions, in Proceedings of the SIGCHI Conference on Human Factors in Computing Systems (ACM, 2014), pp. 1439-1448.

[41] A. Pauchet, N. Chaignaud and A. El Fallah Seghrouchni, A computational model of human interaction and planning for heterogeneous multi-agent systems, in Proceedings of the 6th International Conference on Autonomous Agents and Multiagent Systems ACM2007, pp. 391-393.

[42] H. Bunt, The DIT ++ taxonomy for functional dialogue markup, in $A A$ $M A S$ Workshop, Towards a Standard Markup Language for Embodied Dialogue Acts2009, pp. 13-24.

[43] E. A. Schegloff and H. Sacks, Opening up closings, Semiotica 8(4) (1973) 289-327.

[44] S. Larsson and D. Traum, Information state and dialogue management in the TRINDI dialogue move engine toolkit, Natural language engineering 6(3\&4) (2000) 323-340.

[45] D. N. Walton and E. Krabbe, Commitment in dialogue: Basic concepts of interpersonal reasoning (NY: State University of New York Press, 1995).

[46] M. P. Singh, Social and psychological commitments in multiagent systems, in AAAI Fall Symposium on Knowledge and Action at Social and Organizational Levels1991, pp. 104-106.

[47] W. Mann, Dialogue macrogame theory, in Proceedings of the 3rd SIGdial Workshop on Discourse and Dialogue2002, pp. 129-141.

[48] T. Yuan, D. Moore and A. Grierson, A human-computer dialogue system for educational debate: A computational dialectics approach, International Journal of Artificial Intelligence in Education 18(1) (2008) 3-26. 
[49] G. Dubuisson Duplessis, V. Letard, A.-L. Ligozat and S. Rosset, Purely Corpus-based Automatic Conversation Authoring, in 10th edition of the Language Resources and Evaluation Conference (LREC) (Portorož, Slovenia, 2016), pp. ISBN: 978-2-9517408-9-1.

[50] M. Odersky, L. Spoon and B. Venners, Programming in Scala: a comprehensive step-by-step guide (Artima Inc, 2008).

[51] S. Larsson, Issue-Based Dialogue Management, Ph.D. dissertation, Department of Linguistics, Goteborg University, (Goteborg, 2002).

[52] J. Cassell and T. Bickmore, Negotiated collusion: Modeling social language and its relationship effects in intelligent agents, User Modeling and UserAdapted Interaction 13(1-2) (2003) 89-132. 